\title{
Neoclassical Viscosity of $L=1$ Helical-Axis Heliotron Plasmas for Arbitrary Collision Frequencies and Radial Electric Fields
}

\author{
Kenji NISHIOKA, Yuji NAKAMURA and Shin NISHIMURA ${ }^{1)}$ \\ Graduate School of Energy Science, Kyoto University, Uji, Kyoto 611-0011, Japan \\ 1) National Institute for Fusion Science, Toki, Gifu 509-5292, Japan
}

(Received 26 November 2013 / Accepted 28 July 2014)

\begin{abstract}
Numerical methods for solving the monoenergetic drift kinetic equation (DKE) are powerful tools for obtaining viscosity coefficients. However, these methods do not apply when the collision frequency and radial electric field become large. For example, when the radial electric field becomes large, poloidal resonance effect occurs and degrades the accuracy of the numerical solutions to DKE. But when we calculate the neoclassical viscosity in Heliotron $\mathrm{J}$, which is an $L=1$ helical-axis heliotron device, we cannot neglect the resonance effect in the presence of high- $Z$ ions. In this study, we combine viscosity coefficients calculated by the numerical method with those obtained from analytical solutions that take the effects of the first poloidal resonance into account. We use this method to obtain monoenergetic viscosity coefficients for arbitrary collision frequencies and radial electric fields in the $L=1$ heliotron device.
\end{abstract}

(C) 2014 The Japan Society of Plasma Science and Nuclear Fusion Research

Keywords: resonance effect, momentum conservation, monoenergetic viscosity coefficients, $L=1$ helical-axis heliotron, drift kinetic equation, radial electric field

DOI: $10.1585 /$ pfr.9.1403145

\section{Introduction}

In nonaxisymmetric toroidal systems, it is one of the crucial works to determine parallel flows, neoclassical viscosities, and ambipolar radial electric fields. To solve the drift kinetic equation (DKE) for neoclassical transport analysis, numerical methods are effective to handle complex magnetic geometries in nonaxisymmetric plasmas. Based on the solution of DKE, neoclassical transport analyses that satisfy parallel momentum conservation between different particle species, self-adjointness, and Galilean invariance have been proposed [1-4] using the monoenergetic viscosity coefficients $L^{*}, M^{*}$, and $G^{(\mathrm{BS})}$, which denote radial particle diffusion, parallel viscosity, and bootstrap current, respectively.

Heliotron $\mathrm{J}$ is a nonaxisymmetric device with an $L=$ $1 / M=4$ helical-axis helical coil, where $L$ and $M$ are the pole and pitch numbers of the helical coil, respectively. Experiments have been performed in this device to change the bumpy component of the magnetic field for investigating the controllability of parallel flow and bootstrap current [5-7]. Furthermore, analyses of the monoenergetic viscosity coefficients obtained from numerical solutions of the DKE have been reported [8]. However, because violations of momentum conservation occur when the collision frequency and radial electric field become large, we often cannot apply numerical methods to evaluate the monoenergetic viscosity coefficients [3,4], numerical methods are inappropriate to solve the DKE. In addition, in the L-H transition phase, poloidal resonance effects de-

author'se-mail: nishioka.kenji.88n@st.kyoto-u.ac.jp grade the accuracy of the numerical monoenergetic viscosity coefficients [9-12] in the large radial electric field limit. When we calculate the neoclassical viscosity of plasmas in Heliotron J, which contain high-Z ions, these effects should be taken into account.

Here, we combine the viscosity coefficients calculated by the numerical method with those from an analytical solution that takes the above effects into account. This enables us to obtain monoenergetic viscosity coefficients for arbitrary collision frequencies and radial electric fields in the $L=1$ heliotron device. We also address the controllability of monoenergetic viscosity coefficients by changing the configuration of the magnetic field.

\section{Analytical Viscosity Coefficients}

The linearized DKE is given by

$$
\begin{aligned}
& \left(V_{\|}+V_{E}\right) f_{a 1}-C_{a}^{\mathrm{L}}\left(f_{a 1}\right) \\
& =-\boldsymbol{v}_{\mathrm{d} a} \cdot \nabla f_{a \mathrm{M}}+\frac{e_{a}}{T_{a}} v_{\|} B \frac{\left\langle B E_{\|}\right\rangle}{\left\langle B^{2}\right\rangle} f_{a \mathrm{M}},
\end{aligned}
$$

where $V_{\|}$is the parallel orbit propagator, $V_{E}$ is the $\boldsymbol{E} \times \boldsymbol{B}$ drift operator, $C_{a}^{\mathrm{L}}$ is the linearized collision operator, $\boldsymbol{v}_{\mathrm{d} a}$ is the radial drift velocity, $f_{a \mathrm{M}}$ is the local Maxwellian distribution function, and $f_{a 1}$ is a distortion from the local Maxwellian [1]. To solve Eq. (1) numerically, we employ the drift kinetic equation solver (DKES) code $[13,14]$. In this code, the pitch-angle scattering (PAS) operator is used as the linearized collision operator for simplicity because it is appropriate when a higher-order Legendre term domi- 
nates. The PAS operator is given by

$$
C_{a}^{\mathrm{PAS}} \equiv \frac{\nu_{\mathrm{D}}^{a}}{2} \frac{\partial}{\partial \xi}\left(1-\xi^{2}\right) \frac{\partial}{\partial \xi},
$$

where $\xi$ is the cosine of the pitch angle of the particle. In Eq. (2), the energy-dependent deflection frequency $v_{\mathrm{D}}^{a}$ is given by

$$
v_{\mathrm{D}}^{a} \equiv \sum_{b} \frac{3 \sqrt{\pi}}{4} \tau_{a b}{ }^{-1} x_{a}{ }^{-3}\left(\Phi\left(x_{b}\right)-G\left(x_{b}\right)\right),
$$

where $(3 \sqrt{\pi} / 4) \tau_{a b}{ }^{-1} \equiv e_{a}^{2} e_{b}^{2} n_{b} \ln \Lambda /\left(4 \pi \varepsilon_{0}^{2} m_{a}^{2} v_{\mathrm{ta}}{ }^{3}\right), \varepsilon_{0}$ is the space permeability, $x_{a} \equiv v / v_{\mathrm{t} a}$ is the particle velocity normalized by the thermal velocity $v_{\mathrm{t} a}=\sqrt{2 T_{a} / m_{a}}$, $\Phi\left(x_{a}\right)$ is the error function, and $G\left(x_{b}\right)$ is the Chandrasekhar function [1-4]. Here, $\tau_{a b}$ is the collision time between test particles of species $a$ and field particles of species $b$. The DKES code solves Eq. (1) by neglecting perpendicular drift terms except for the $\boldsymbol{E} \times \boldsymbol{B}$ drift in $\boldsymbol{v}_{\mathrm{d} a} \cdot \nabla f_{a 1}$. In nonaxisymmetric stellarator/heliotron configurations under low collisionality conditions, a so-called bounce-averaged perturbation is generated in the ripple-trapped pitch-angle range. Therefore, retaining only $V_{\|} f_{a 1}$ as the derivative of $f_{a 1}$ is usually inadequate. To include the poloidal precession of trapped particles, $V_{E} f_{a 1}$ is also retained. In addition to this drift due to the radial electric field, which is known as the superbanana diffusion regime, bounce-averaged perpendicular drifts due to $\nabla B$ and the magnetic field curvature $\boldsymbol{b} \cdot \nabla \boldsymbol{b}$ are sometimes not negligible in conditions of weak radial electric field strengths and low collision frequencies. For stellarator/heliotron magnetic fields

$$
\begin{aligned}
B / B_{0}= & 1-\varepsilon_{\mathrm{t}}(r) \cos \theta+\varepsilon_{\mathrm{h}}(r, \theta) \\
& \times \cos (L \theta-M \zeta+\gamma(r, \theta)),
\end{aligned}
$$

radial diffusion in the superbanana collisionality regime [15] is estimated to be

$$
L^{*(\mathrm{SB})}=4 \sqrt{\pi} \frac{\varepsilon_{\mathrm{h}} \sqrt{\varepsilon_{\mathrm{t}}}}{\left(\mathrm{d} \varepsilon_{\mathrm{h}} / \mathrm{d} r\right)^{2}}\left(\frac{e_{a}}{m_{a} v}\right)^{2} \frac{v_{\mathrm{D}}^{a}}{v},
$$

for the collisionality condition $L^{*(\mathrm{SB})}<L^{*(1 / v)}$. Here, the flux function $r=a \sqrt{s}$ is the effective minor radius, which is defined by the normalized toroidal flux $s$ and the effective plasma radius $a=0.16 \mathrm{~m}$. The $1 / v$ diffusion $L^{*(1 / v)}$ can be estimated by the well-known Shaing-Hokin formula [16],

$$
L^{*(1 / v)}=\frac{4 \sqrt{2}}{9 \pi} \frac{\varepsilon_{\mathrm{t}}^{2} \varepsilon_{\mathrm{h}}^{3 / 2}}{\left(\psi^{\prime}\right)^{2}} \frac{v}{v_{\mathrm{D}}^{a}},
$$

where $\psi$ is a toroidal magnetic flux divided by $2 \pi$, and $\psi^{\prime}=\mathrm{d} \psi / \mathrm{d} r$. An empirical scaling for radial diffusion in the collisionless detrapping collisionality regime, which is suppressed by $\boldsymbol{E} \times \boldsymbol{B}$ drift [17], is

$$
L^{*(\mathrm{CD})}=\frac{\sqrt{\pi}}{6} \varepsilon_{\mathrm{t}}^{3 / 2} \frac{v_{\mathrm{D}}^{a}}{v}\left(\frac{E_{r}}{v}\right)^{-2},
$$

where $E_{r}=-\partial \Phi_{\mathrm{E}} / \partial r$, and $\Phi_{\mathrm{E}}(r)$ is the electrostatic potential. In a rough estimate of the $\left(v_{\mathrm{D}}^{a}, E_{r}\right)$ space region in which $\nabla B$ and curvature drifts in the poloidal direction are not negligible, the poloidal dependence of $\varepsilon_{h}$ is neglected, at least in these asymptotic limit formulas for $L^{*(\mathrm{SB})}, L^{*(1 / v)}$, and $L^{*(\mathrm{CD})}$. Therefore the bounce-averaged poloidal drift of the trapped particles due to $\mathrm{d} \varepsilon_{h} / \mathrm{d} r$ is not negligible compared with that due to $E_{r}$ in conditions of weak radial electric fields of

$$
\left|E_{r}\right|<\frac{1}{2 \sqrt{6}}\left(\frac{\varepsilon_{\mathrm{t}}}{\varepsilon_{\mathrm{h}}}\right)^{1 / 2}\left|\frac{\mathrm{d} \varepsilon_{\mathrm{h}} / \mathrm{d} r}{e_{a}}\right| m_{a} v^{2},
$$

and low collision frequencies of

$$
v_{D}^{a}<\frac{2^{1 / 4}}{3 \pi^{3 / 4}}\left(\varepsilon_{\mathrm{t}}^{3 / 2} \varepsilon_{\mathrm{h}}^{1 / 2}\right)^{1 / 2}\left|\frac{\mathrm{d} \varepsilon_{\mathrm{h}} / \mathrm{d} r}{e_{a} \psi^{\prime}}\right| m_{a} v^{2} .
$$

For hydrogen and deuterium ions in typical Heliotron $\mathrm{J}$ experimental conditions $\left(\mathrm{d} \varepsilon_{\mathrm{h}} / \mathrm{d} r \simeq 2 \varepsilon_{\mathrm{h}} / r \approx 4 \mathrm{~m}^{-1}, \varepsilon_{\mathrm{t}} \approx \varepsilon_{\mathrm{h}} \approx\right.$ $0.1, \psi^{\prime} \approx 0.1 \mathrm{Tm}, m_{a} v^{2} \approx 100 \mathrm{eV}$ ), Eqs. (8) and (9) are estimated to be $\left|E_{r}\right|<80 \mathrm{~V} / \mathrm{m}$ and $v_{D}^{a}<70 \mathrm{~s}^{-1}$, respectively, and thus they are negligibly smaller than the actual ambipolar electric field strength (few $\mathrm{kV} / \mathrm{m}$ ) and collision frequency $\left(10^{5} \mathrm{~s}^{-1}\right)$. Therefore, neglect of $\nabla B$ and curvature drifts in the poloidal direction in Eq. (1) is adequate for studies at Heliotron $\mathrm{J}$ conditions.

In the DKES, the monoenergetic transport coefficients $D_{j k}(K)$ are defined by

$$
D_{j k}(K) \equiv\left(\sigma_{j}^{+}, F_{k}^{+}\right)+\left(\sigma_{j}^{+}, F_{k}^{-}\right) \quad(j, k=1,3),
$$

where $K \equiv x_{a}{ }^{2} \equiv m_{a} v^{2} / 2 T_{a}$ is the normalized kinetic energy. In Eq. (10), $\sigma_{1}^{+}$and $\sigma_{3}^{+}$are source terms associated with the radial particle flux and parallel particle flow, respectively, and $F_{j}^{+}$and $F_{j}^{-}$are the response functions associated with $\sigma_{j}^{+}$. These functions are defined by

$$
\begin{aligned}
& \left(V_{\|}+V_{E}\right) F_{j}^{-}-C_{a}^{\mathrm{L}} F_{j}^{+} \equiv \sigma_{j}^{+} \quad(j=1,3) . \\
& \left(V_{\|}+V_{E}\right) F_{j}^{+}-C_{a}^{\mathrm{L}} F_{j}^{-} \equiv 0
\end{aligned}
$$

The quantity $(\mathcal{F}, \mathcal{G})$ represents the inner product

$$
(\mathcal{F}, \mathcal{G}) \equiv \frac{1}{2} \int_{-1}^{1} \mathrm{~d} \xi\langle\mathcal{F} \mathcal{G}\rangle,
$$

of arbitrary functions $\mathcal{F}(\theta, \zeta, \xi)$ and $\mathcal{G}(\theta, \zeta, \xi)$. We obtain $D_{j k}(K)$ from the inner product of $\sigma_{j}^{+}$and $\left(F_{k}^{+}+F_{k}^{-}\right)$by applying a variational principle for the Fourier-Legendre expanded distribution function. This $D_{j k}(K)$ depends on the magnetic field configuration, $v_{\mathrm{D}}^{a} / v$ and $E_{r} / v$. Numerical analyses of neoclassical transport by use of these monoenergetic viscosity coefficients have been performed [1-4]. In these methods, $g_{a}=f_{a 1}-f_{a 1}^{(l=1)}$ was introduced. The $l=1$ Legendre component $f_{a 1}^{(l=1)}$ of the distribution function $f_{a 1}$ is associated with parallel particle flow. This $g_{a}$ is determined by the driving forces $G_{X a}$ from the radial gradient and $G_{U a}$ from the parallel flow [1]. The $G_{X a}$ and $G_{U a}$ are obtained from the differential equations

$$
\left(V_{\|}+V_{E}-C_{a}^{\mathrm{L}}\right)\left[\begin{array}{c}
G_{U a} \\
G_{X a}
\end{array}\right]=\left[\begin{array}{c}
\sigma_{U a} \\
\sigma_{X a}
\end{array}\right],
$$



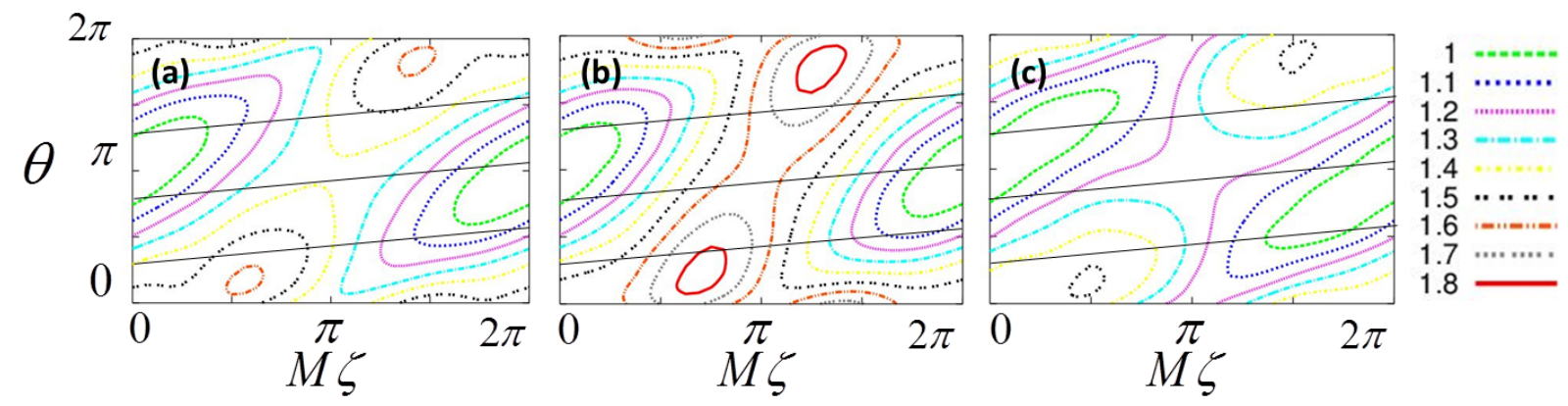

Fig. 1 Contour maps of $|\boldsymbol{B}|$ in the $(\theta, \zeta)$ plane on the flux surface $r / a=0.71$ for (a) STD configuration, (b) HG configuration, and (c) RG configuration. Black solid lines denote magnetic field lines.

where $\sigma_{U a}$ and $\sigma_{X a}$ are defined in Ref. [1]. Note the following about the approximation for $V_{E}$ used in the DKES code. As shown in Ref. [13], a straightforward derivation of $V_{E}$ gives

$$
\begin{aligned}
V_{E}= & E_{r} \frac{\nabla r \times \boldsymbol{B}}{B^{2}} \cdot \nabla+E_{r}\left(\frac{\nabla r \times \boldsymbol{B}}{2 B^{2}} \cdot \nabla \ln B\right) \\
& \times\left\{\left(1-\xi^{2}\right) \xi \frac{\partial}{\partial \xi}+\left(1+\xi^{2}\right) v \frac{\partial}{\partial v}\right\} .
\end{aligned}
$$

For the purpose to operate $G_{U a}$ and $G_{X a}$, which are dominated by poloidal and toroidal variations of higher Legendre orders $l \geq 2$, the second term is a higher order of $\delta B$ when comparing with the first term. Here, $\delta B \equiv B-\langle B\rangle$ is the magnetic field strength modulation on the surfaces. A primary advantage of omitting this order together with the use of $C_{a}^{\mathrm{PAS}}$ is a reduction in the phase-space dimension of Eq. (13) from $4 \mathrm{D}(\theta, \zeta, v, \xi)$ to $3 \mathrm{D}(\theta, \zeta, \xi)$. In this $3 \mathrm{D}$ calculation, the drift velocity $\boldsymbol{v}_{E}=\boldsymbol{E} \times \boldsymbol{B} / B^{2}$ is replaced by $\boldsymbol{E} \times \boldsymbol{B} /\left\langle B^{2}\right\rangle$ for retaining the surface-averaged conservation of particles and energy $\left\langle\int_{-1}^{1}\left(V_{E} \mathcal{F}\right) \mathrm{d} \xi\right\rangle=0$ and the antisymmetric property of the Vlasov operator $\left(\mathcal{G}, V_{E} \mathcal{F}\right)=\left(\mathcal{F}, V_{E} \mathcal{G}\right)$, which is required along with the self-adjoint property of the collision operator for obtaining the Onsager symmetric full transport matrix.

If

$$
e_{a} \sigma_{1}^{+} \gg m_{a} v_{\mathrm{D}}^{a}\left|V_{E} \int^{l} \tilde{U} \mathrm{~d} l\right|
$$

is satisfied, we can calculate $G_{X a}$ using $D_{j k}(K)$ obtained from the DKES $[3,4,18]$, where $\int^{l} \mathrm{~d} l$ denotes the integral along the magnetic field line and $\tilde{U}$ is obtained as the solution of

$$
\boldsymbol{B} \cdot \nabla\left(\frac{\tilde{U}}{B}\right)=\boldsymbol{B} \times \nabla s \cdot \nabla\left(\frac{1}{B^{2}}\right) .
$$

However, in the large $v_{\mathrm{D}}^{a} E_{r} / v^{2}$ limit, Eq. (15) cannot be satisfied and conservation of momentum is violated. In this case, $D_{11}$ and $D_{13}=D_{31}$ calculated by the DKES cannot be used to obtain $L^{*}$ and $G^{(\mathrm{BS})}$. Moreover, in the large $v_{\mathrm{D}}^{a} / v$ limit, the monoenergetic viscosity coefficients are in- versely proportional not to $v_{\mathrm{D}}^{a}$ but to $v_{\mathrm{T}}^{a}[1-4]$, where

$$
\begin{aligned}
& v_{\mathrm{T}}^{a}=3 v_{\mathrm{D}}^{a}+v_{\mathrm{E}}^{a} \\
& =\left(\frac{3 \sqrt{\pi}}{4}\right) \sum_{b} \tau_{a b}{ }^{-1}\left\{\frac{H\left(x_{b}\right)}{x_{a}{ }^{3}}+4 \frac{T_{a}}{T_{b}}\left(1+\frac{m_{b}}{m_{a}}\right) \frac{G\left(x_{b}\right)}{x_{a}}\right\},
\end{aligned}
$$

represents a collision frequency for relaxation of pressure anisotropy. In Eq. (17), $v_{\mathrm{E}}^{a}$ is the energy exchange frequency and $H\left(x_{b}\right)=\Phi\left(x_{b}\right)-3 G\left(x_{b}\right)$. Furthermore, when $E_{r}$ becomes sufficiently large, the poloidal velocities of the passing particles are canceled by the poloidal components of $\boldsymbol{v}_{\mathrm{E}}$. In such a case, particles do not describe banana orbits. This poloidal resonance occurs at $M_{\mathrm{p} a} \sim|m-n q| / m$ for each $(m, n)$ Fourier mode and reduces $D_{11}$ significantly, where $M_{\mathrm{p} a}=\left|\boldsymbol{v}_{\mathrm{E}}\right| B / v_{\mathrm{t} a} B_{\mathrm{p}}$ is the poloidal Mach number, $q$ is the safety factor, and $B_{\mathrm{p}}$ is the poloidal magnetic field [10-12]. For the $(1,0)$ Fourier mode, resonance occurs at $M_{\mathrm{p} a} \sim 1$. This effect is closely related to the L-H transition theory, however, this topic is beyond the scope of this study. Therefore, we adopt the analytical solution that includes poloidal resonance in the $v_{\mathrm{D}}^{a} / v$ and $E_{r} / v$ regime for which the numerical method is inappropriate. In the plateau regime, we can obtain the viscosity coefficients using the Krook operator and Fourier expansion. For each Fourier component, we define $b_{m n}$ as

$$
b_{m n} \equiv \frac{E_{r}}{v} \frac{\left(B_{\zeta} m+B_{\theta} n\right)}{\left(\chi^{\prime} m-\psi^{\prime} n\right)}\left\langle B^{2}\right\rangle^{-\frac{1}{2}}
$$

where $\chi$ is a poloidal magnetic flux divided by $2 \pi$. For $\left|b_{m n}\right|<3.0 \times 10^{-2}$, the monoenergetic viscosity coefficients in the plateau and Pfirsch-Schlüter regimes are given in Ref. [19]. For the condition $\left|b_{m n}\right|>3.0 \times 10^{-2}$, we replace the function

$$
\left\{\left(\frac{8}{\pi}\left|\chi^{\prime} m-\psi^{\prime} n\right|\right)^{3 / 2}+\left(5 \frac{\nu_{\mathrm{T}}^{a}}{v} \sqrt{\left\langle B^{2}\right\rangle} \frac{V^{\prime}}{4 \pi^{2}}\right)^{3 / 2}\right\}^{-2 / 3},
$$

in Eqs. (17) and (18) in Ref. [19] by

$$
\frac{9 a_{m n}}{8\left|\chi^{\prime} m-\psi^{\prime} n\right|} \int_{-1}^{1} \frac{\left(\xi^{2}-1 / 3\right)^{2}}{\left(\xi-b_{m n}\right)^{2}+a_{m n}^{2}} \mathrm{~d} \xi
$$




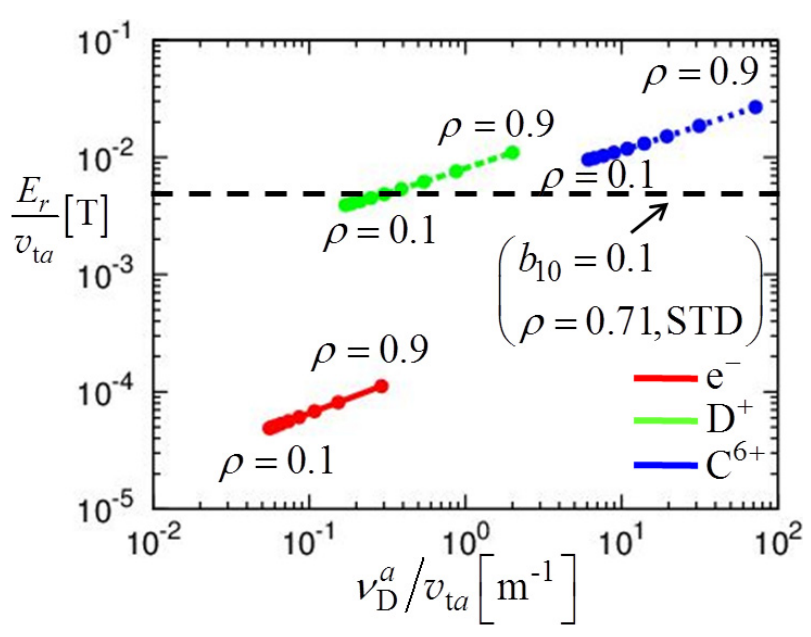

Fig. 2 Typical $v_{\mathrm{D}}^{a}\left(v=v_{\mathrm{t} a}\right) / v_{\mathrm{t} a}$ and $E_{r} / v_{\mathrm{t} a}$ for all particle species on the $\rho=0.1-0.9$ magnetic flux surface in Heliotron $\mathbf{J}$ plasma, where $\rho=r / a$. Plasma particle parameters are as follows: $E_{r}=0.5 \mathrm{kV} / \mathrm{m}, n_{\mathrm{e}}(\rho)=1.5 \times 10^{19}\left(1-\rho^{2}\right) \mathrm{m}^{-3}$, $T_{\mathrm{e}}(\rho)=300\left(1-\rho^{2}\right) \mathrm{eV}, T_{I}(\rho)=175\left(1-\rho^{1.57}\right)^{1.11} \mathrm{eV}$, and $Z_{\text {eff }}=1.9$.

where $a_{m n}$ is defined by

$$
a_{m n} \equiv \frac{v_{\mathrm{T}}^{a}}{v} \frac{\left\langle B^{2}\right\rangle^{1 / 2}}{\left|\chi^{\prime} m-\psi^{\prime} n\right|} \frac{V^{\prime}}{4 \pi^{2}} .
$$

The analytical formula (for the single Fourier mode $m=1$, $n=0$ ) is compared with the numerical solution (DKES) in Ref. [12]. Note that Eq. (20) is inapplicable for calculations in the weak $E_{r}$ limit because the conservation of particles and of energy are violated.

\section{Numerical Results}

The monoenergetic viscosity coefficients were studied for three magnetic configurations: standard magnetic ripple configuration (STD), high-bumpy configuration (HG), and reversed-mirror ripple configuration $(\mathrm{RG})$. Contour maps for $|\boldsymbol{B}|$ on the flux surface of $r / a=0.71$ are shown in Fig. 1. The figure shows that the largest ripple depth occurred in the HG configuration and the smallest occurred in the RG configuration. Figure 2 shows typical $v_{\mathrm{D}}^{a} / v_{\mathrm{t} a}$ and $E_{r} / v_{\mathrm{t} a}$ for $\mathrm{e}^{-}, \mathrm{D}^{+}$, and $\mathrm{C}^{6+}$ in Heliotron $\mathrm{J}$. The figure shows that $b_{10}>0.1$ is satisfied for $\mathrm{C}^{6+}$. This indicates a strong reduction in monoenergetic transport coefficients by the first poloidal resonance in the presence of $\mathrm{C}^{6+}$ ions in Heliotron J. Therefore, when we estimate the monoenergetic viscosity coefficients for such plasmas, we should include the poloidal resonance effect. The monoenergetic viscosity coefficients $L^{*}, M^{*}$, and $G^{(\mathrm{BS})}$ obtained from analytical and numerical methods in the STD configuration are shown in Fig. 3. For $M^{*}$ and $G^{(\mathrm{BS})}$, we connect the analytical and numerical solutions at the point where the particle velocity gives the maximum value of $M^{*}$ obtained from the DKES. We connect $L^{*}$ at the point where the particle
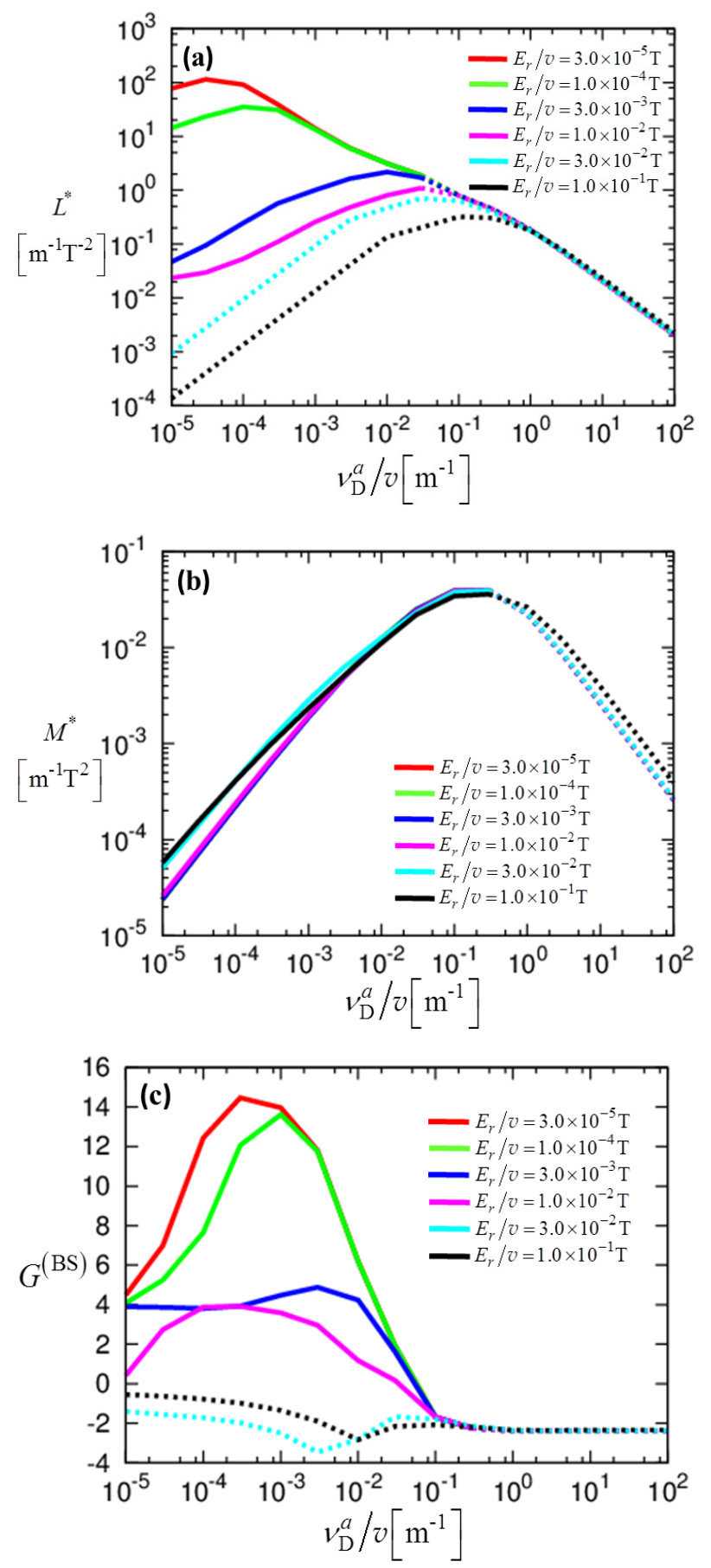

Fig. 3 Monoenergetic viscosity coefficients $L^{*}, M^{*}$, and $G^{(\mathrm{BS})}$ on the flux surface $r / a=0.71$ in the STD configuration. Solid lines indicate numerical solutions from the DKES and dotted lines indicate analytical solutions.

velocity satisfies

$$
\frac{v_{\mathrm{T}}^{a}}{v}=\frac{8}{5} \frac{4 \pi}{V^{\prime}} \chi^{\prime}\left\langle B^{2}\right\rangle^{-1 / 2}
$$

For simplicity, here we replace $v_{\mathrm{T}}^{a}$ by $3 v_{\mathrm{D}}^{a}$. Figure 3 shows that using this method, we can obtain smooth and continuous monoenergetic viscosity coefficients at arbitrary collision frequencies and electric fields. Figure 4 shows the de- 

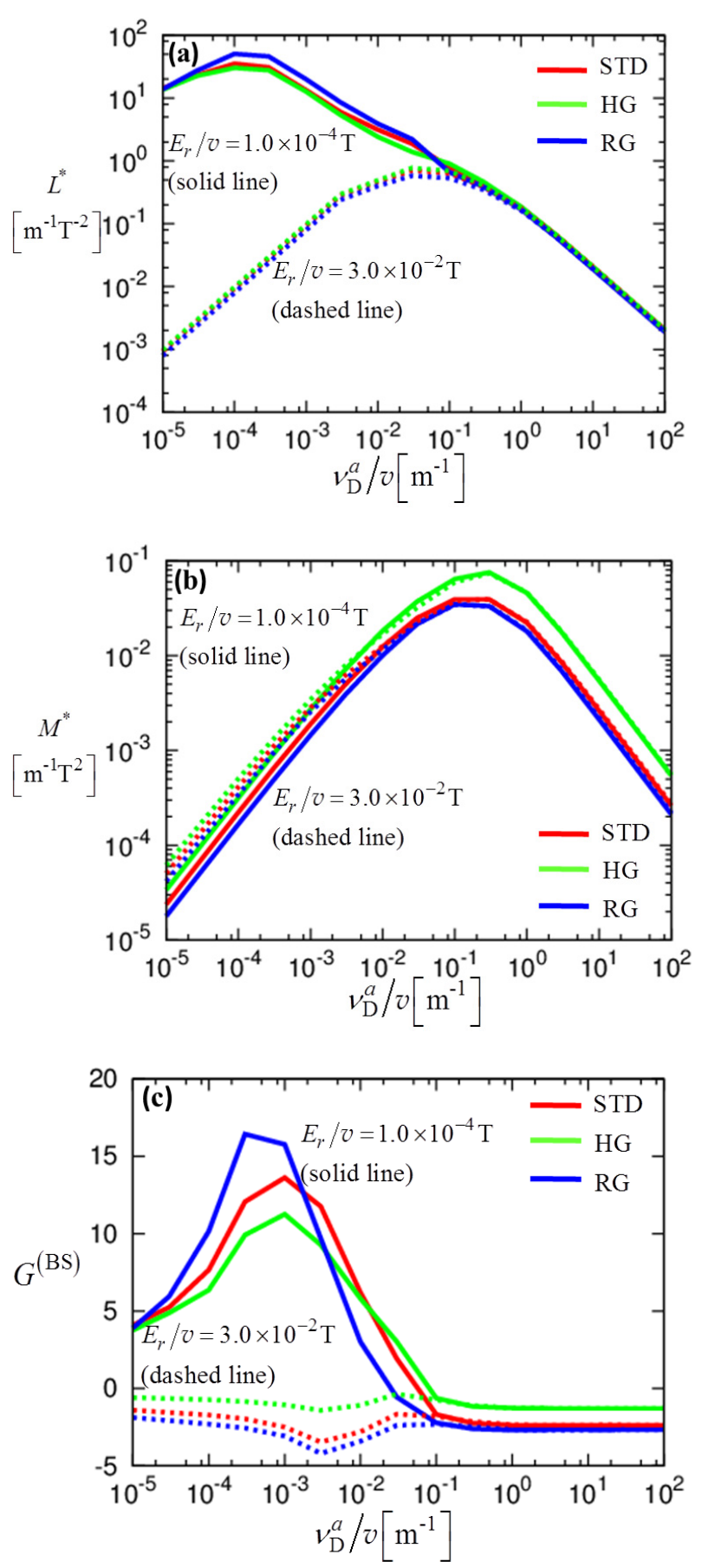

Fig. 4 Monoenergetic viscosity coefficients at weak $\left(E_{r} / v=\right.$ $\left.1.0 \times 10^{-4} \mathrm{~T}\right)$ and strong $\left(E_{r} / v=3.0 \times 10^{-2} \mathrm{~T}\right)$ electric field on the flux surface $r / a=0.71$ in the HG, STD and RG configurations.

pendence of the bumpy component on the monoenergetic viscosity coefficients at weak $\left(E_{r} / v=1.0 \times 10^{-4} \mathrm{~T}\right)$ and strong $\left(E_{r} / v=3.0 \times 10^{-2} \mathrm{~T}\right)$ electric fields. In the HG configuration, $M^{*}$ is larger than those in the other two configurations in all collisionality regimes. Since $M^{*}$ is closely associated with parallel viscosity damping, this result shows that the large magnetic ripple depth by the bumpy component in the HG configuration yields strong viscosity damping. Parallel particle flows and bootstrap currents are expected to be suppressed in this configuration. However, a detailed analysis of parallel particle flows using the moment method [1-4] is beyond the scope of this study.

\section{Conclusions}

In this study, we estimated the monoenergetic viscosity coefficients of plasmas in an $L=1$ helical-axis heliotron device, Heliotron J. We applied analytic calculations together with a numerical solution to avoid problems concerning the validity of the simplified DKE in the DKES code due to the poloidal resonance effect and violation of parallel momentum conservation. The combination of numerical solutions from the DKES code with analytical solutions allowed us to obtain continuous monoenergetic viscosity coefficients for arbitrary collision frequencies and electric fields. We found that changes in the bumpy field component can effectively control the neoclassical viscosity. A detailed analysis of the parallel particle flows using the moment method will be reported in future work.

\section{Acknowledgments}

One of the authors (K.N) would like to thank Heliotron J experimental group and Dr. H. Lee of Natural Science Research Institute, Korea Advanced Institute of Science and Technology for fruitful discussions. This research was supported by the NIFS for No.NIFSKUHL038.

[1] H. Sugama and S. Nishimura, Phys. Plasmas 9, 4637 (2002).

[2] H. Sugama and S. Nishimura, Phys. Plasmas 15, 042502 (2008).

[3] S. Nishimura et al., Phys. Plasmas 17, 082510 (2010).

[4] S. Nishimura et al., Phys. Plasmas 18, 069901 (2011).

[5] H. Lee et al., Plasma Phys. Control. Fusion 55, 035012 (2013).

[6] S. Kobayashi et al., D1-5-O4, The 12th Asia Pacific Physics Conference of AAPPS, Japan, 2013.

[7] G. Motojima et al., Nucl. Fusion 47, 1045 (2007).

[8] S. Nishimura et al., J. Plasma Fusion Res. 8, 1003 (2009).

[9] K.C. Shaing and E.C. Crume, Jr., Phys. Rev. Lett. 63, 2369 (1989).

[10] K.C. Shaing, Phys. Fluids B 5, 3841 (1993).

[11] J. Lore et al., Phys. Plasmas 17, 056101 (2010).

[12] H. Maaßberg et al., Phys. Fluids B 5, 3627 (1993).

[13] S.P. Hirshman et al., Phys. Fluids 29, 2951 (1986).

[14] W.I. van Rij and S.P. Hirshman, Phys. Fluids B 1, 563 (1989).

[15] D.E. Hastings et al., Nucl. Fusion 29, 2951 (1985).

[16] K.C. Shaing and S.A. Hokin, Phys. Fluids 26, 2136 (1983).

[17] E.C. Crume, Jr. et al., Phys. Fluids 31, 11 (1988).

[18] D.A. Spong, Phys. Plasmas 12, 056114 (2005).

[19] S. Nishimura et al., Fusion Sci. Technol. 51, 61 (2007). 